\title{
A rate study of Type Ia supernovae with Subaru/XMM-Newton Deep Survey
}

\author{
Yutaka Ihara, Mamoru Doi, Tomoki Morokuma, Raynald Pain, \\ Naohiro Takanashi, Naoki Yasuda, Greg Aldering, Kyle Dawson, \\ Gerson Goldhaber, Isobel Hook, Chris Lidman, Saul Perlmutter, \\ Anthony Spadafora, Nao Suzuki and Lifan Wang (for the Supernova \\ Cosmology Project Collaboration)
}

\begin{abstract}
We present a measurement of the rate of high-z Type Ia supernovae (SNe Ia) using multi-epoch observations of Subaru/XMM-Newton Deep Field (SXDF) with Suprime-Cam on the Subaru Telescope. Although SNe Ia are regarded as a standard candle, progenitor systems of SNe Ia have not been resolved yet. One of the key parameters to show the progenitor systems by observations is the delay time distribution between the binary system formation and subsequent SN explosion. Recently, a wide range of delay time is studied by SN Ia rates compared with an assumed cosmic star formation history. If SNe Ia with short delay time are dominant, the cosmic SN Ia rate evolution should closely trace that of the cosmic star formation. In order to detect a lot of high-z SNe Ia and measure SN Ia rates, we repeatedly carried out wide and deep imaging observations in the $i^{\prime}$-band with Suprime-Cam in 2002 (FoV $\sim 1 \mathrm{deg}^{2}, m_{i}<25.5 \mathrm{mag}$ ). We obtained detailed light curves of the variable objects, and 50 objects are classified as SNe Ia using the light curve fitting method at the redshift range of $0.2<z<1$. In order to check the completeness and contamination of the light curve classification method, we performed Monte Carlo simulations and generated $\sim 100,000$ light curves of SNe Ia and II from templates. The control time and detection efficiency of the SN survey are also calculated using the artificial light curves. We derived an increasing trend of rates at around $z \sim 1.2$. Our results are almost consistent with other SN Ia rate results from low-z to high-z. Our results are the first results of high-z SN Ia rates with large statistics using light curves obtained by ground based telescopes, and give us visions of the SN rate studies for the future.
\end{abstract}

\section{Motivation}

Studying the rate of Type Ia supernovae (SNe Ia) is very important to understand the progenitor of SNe Ia. The dependence of SN Ia rates on properties of host galaxies indicates a wide range of the delay time between the binary system formation and subsequent SN explosion, from $\leqslant 1$ Gyr to $\sim 10$ Gyr (Mannucci et al. 2006). Recently, a more quantitative measurement of the delay time distribution (DTD) of SNe Ia has been made by Totani et al. (2008), and DTD is well described as $\propto t^{-1}$ in $0.1-10 \mathrm{Gyr}$, confirming that DTD extends to short delay time of $\leqslant 0.1$ Gyr. Because of the existence of short delay time SNe Ia, it is predicted that the cosmic SN Ia rate evolution should closely trace that of the cosmic star formation, and hence SN Ia rates should be increasing with redshift toward the peak of the cosmic SFR at $z \sim 2-3$ (e.g., Hopkins \& Beacon 2006). Measuring high-z $(z>1.0)$ SN Ia rates is very difficult because SNe Ia are too faint $\left(m_{i}>25 \mathrm{mag}\right)$, and we need 8-m class telescopes or the Hubble Space Telescope (HST). Dahlen et al. (2008) (here after D08) reported the highest-z SN Ia rates with HST. Interestingly, their SN Ia rates show a decrease beyond $z \sim 1.6$, which is in contradiction with the expectation from the DTD measurements. However, the D08 rates have large 
errors because of small statistics in the highest redshift bin. Poznanski et al. 2007 (here after P07) showed similar decreasing rate using Subaru Deep Field (SDF) data, but the uncertainty is still large. Therefore it is important to measure accurate high-z SN Ia rates using 8-m class telescopes for long time.

\section{Observations}

The Subaru/XMM-Newton Deep Survey (SXDS) is a multiwavelength survey from Xray to radio. The survey targets a $1.22 \mathrm{deg}^{2}$ field centered on $(02 \mathrm{~h} 18 \mathrm{~m} 00 \mathrm{~s},-05 \mathrm{~d} 00 \mathrm{~m} 00 \mathrm{~s})$, hereafter referred to as the Subaru/XMM-Newton Deep Field (SXDF). The optical imaging component of the survey was carried out using Suprime-Cam on the Subaru 8.2-m telescope, starting in Sep. 2002. In order to detect and follow the lightcurves of optically faint variable objects, the Suprime-Cam observations were split into exposures of 1800 7200 seconds separated by periods of days to weeks. Between Sep. 2002 and Dec. 2002, the fields were observed 5-7 times in the $i^{\prime}$-band and 2-4 times in the $R_{c}$ and $z^{\prime}$-bands. After the 2002 observations finished, we took reference images in the $i^{\prime}$-band in 2003 and 2005, in the $z^{\prime}$-band in 2005, and in the $R_{c^{-}}$band in 2008. In addition, Follow-up spectroscopic observations were carried out during the variable object survey in 2002 to obtain types and redshifts of likely SN candidates using 8-10m class telescopes, such as Subaru, Gemini-N, Keck, and VLT.

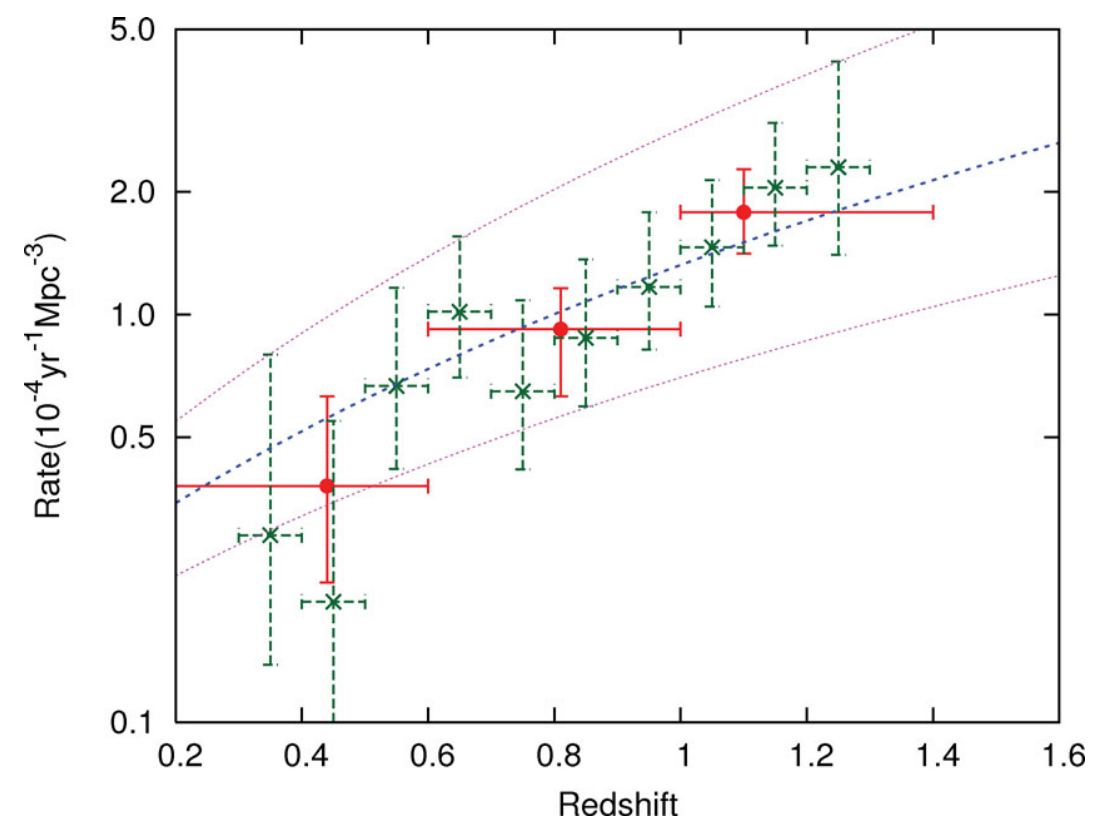

Figure 1. SN Ia rates in SXDF. Red circles show the rates with $\Delta z=0.4$ redshift bin, which is the same bin width as Dahlen et al. 2008. Green crosses are the rates with $\Delta z=0.1$ redshift bin, which is used to perform the power law fitting. The blue dashed line shows $\mathrm{r}_{V}(\mathrm{z})=\mathrm{r}_{o}(1+\mathrm{z})^{\alpha}$, where $\mathrm{r}_{o}=0.21_{0.06}^{0.09+0.04} \times 10_{-0 .}^{-4} \mathrm{yr}^{-1} \mathrm{Mpc}^{-3}, \alpha=2.63_{-0.44-0.25}^{+0.60+0.10}$. The rates are increasing toward high redshift end. 


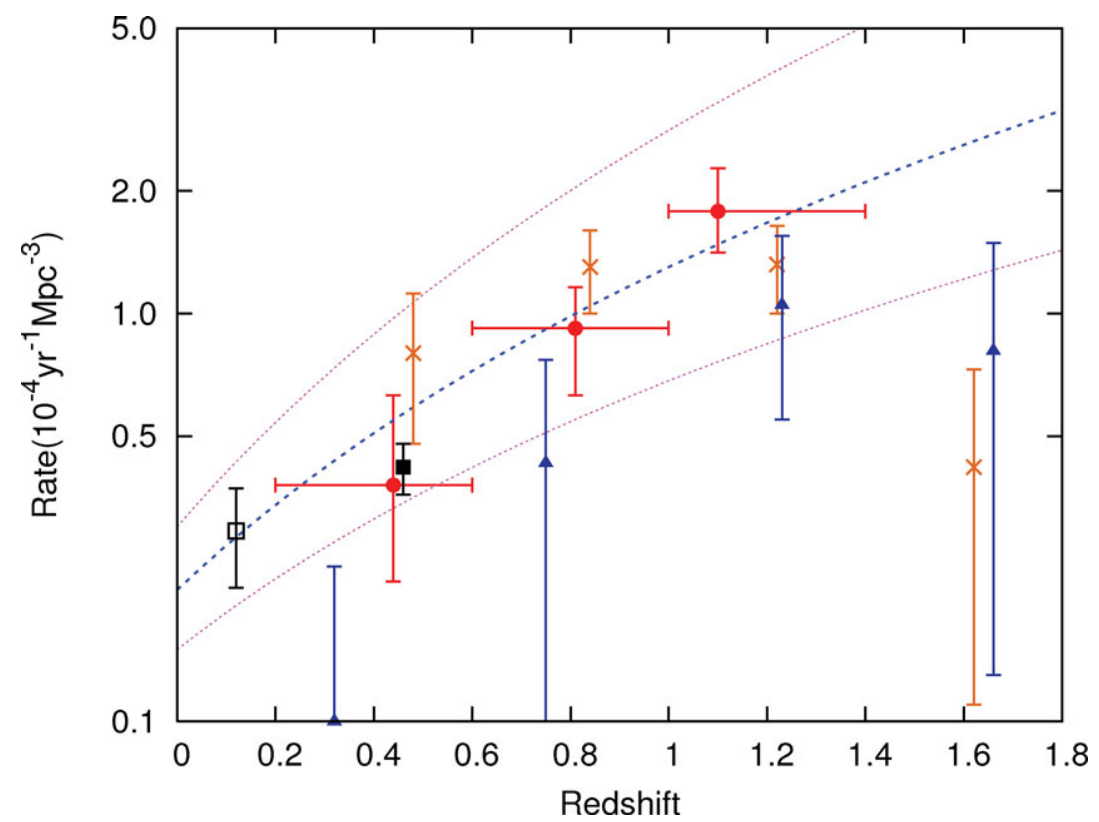

Figure 2. Comparison with the various previous works and our results. The red circles show our results. The orange crosses are the results by Dahlen et al. 2008. The blue open triangles are the results by Poznanski et al. 2007. The black open square is the result at $\mathrm{z}=0.12$ by Dilday et al. 2008. The black square is the result of Neill et al. 2006.

\section{Rate calculation}

We detected 1040 variable objects in the SXDS, and they include variable stars, AGNs, and SNe. We select SNe Ia from all of the variable objects using lightcurves and colors. First, we use long term variability to remove AGN and variable stars from the initial sample of 1040 variable objects. Since SNe should show variabilities for a few months time scale and we select SNe occurred in 2002, the objects showing variabilities (above $5 \sigma$ ) in 2003 or 2005 are removed from our SN sample. In order to avoid false detection, We also require that SN candidates show at least a $5 \sigma$ increase in 3 or more epochs in the $i^{\prime}$-band. After this stage, 192 variable objects remain as SN candidates. Second, we discriminate Type II SNe using $i^{\prime}$-band lightcurves with the $\chi^{2}$ fitting method. The Type Ia template in this fitting is constructed by Hsiao et al. (2007) and Type II templates are condtructed by 12 observed Type II SNe (e.g. SDSS-II SN survey / Frieman et al. 2008). After this stage, 50 variable objects remain as SN Ia candidates. Since it is impossible to classify the candidates into SNe Ia and Ib/c by only lightcurves because the lightcurves of SNe Ia and $\mathrm{Ib} / \mathrm{c}$ are similar, we discriminate $\mathrm{SNe} \mathrm{Ib} / \mathrm{c}$ from SNe Ia using color information of Rc- $i^{\prime}$ vs $i^{\prime}-z^{\prime}$. But only 15 SN Ia candidates have the color information. Hence we estimate the ratio of the contamination of $\mathrm{SNe} \mathrm{Ib} / \mathrm{c}$ using the samples having the color information. In order to estimate the completeness and the contamination of the lightcurve classification, we generate many artificial SNe with the Monte Carlo simulation based on time sampling and observation depth of our SXDS variable object survey.

On the other hand, We compute the control time as a function of redshift. The control time is the time interval during which we can detect the SN explosion, and the time is defined in an observed frame. We calculate how many days the artificial SNe Ia can be observed. The observing epochs and detection limits are set to be the same as the 
SXDS variable object survey, and we measure the observable time duration of individual artificial SN Ia lightcurves.

Given the supernova rate per unit comoving volume $r_{V}(z)$, the average number of SNe we expect to observe in the redshift bin $\left[z_{1}, z_{2}\right]$ is given by

$$
N_{\exp }\left(z_{1}<z<z_{2}\right)=\int_{z_{1}}^{z_{2}} \frac{r_{V}(z)}{1+z} C T(z) V(z) d z,
$$

where $V(z) d z$ is the comoving volume in a redshift slice of width $d z$ and $C T(z)$ is the observer frame "control time". The results are shown in Fig. 1.

\section{Discussion}

Our results and results of previous studies are plotted in Fig. 2. In the low redshift range, the fitted rate function is well consistent with the result at $z=0.12$ by Dilday et al. (2008). In the middle redshift range, Neill et al. (2006) at $\mathrm{z}=0.47$ is also consistent with our results, though our results in the mid redshift range have larger errors than the results of Neill et al. (2006) due to small statistics. At high redshift range, we can compare two observational results of SN Ia rates, which are by Da08 and by Po07. The $\mathrm{SN}$ Ia rates by Da08 show flat tendency from $\mathrm{z} \sim 0.8$ to $\mathrm{z} \sim 1.2$, and then the highest rate at $\mathrm{z} \sim 1.6$ was lower than the rates at $\mathrm{z} \sim 0.8-1.2$. Our results show the SN Ia rates are increasing toward $\mathrm{z} \sim 1.2$. The results of $\mathrm{Da} 08$ and our results seem to show the different rate tendency, but they might be in statistical agreement. Po07 showed that the rates were increasing until $\mathrm{z} \sim 1.2$ and then decreasing at the highest redshift $\mathrm{z} \sim 1.6$. The rate tendency of $\mathrm{Po} 07$ is consistent with out results, but the values of the rates of Po07 were slightly lower than those of our results at $\mathrm{z}<1.0$. The reason for the difference is possibly that the uncertainty of both results is quite large due to small statistics.

Our survey is optimized for $\mathrm{z}<1.4$ given the rest frame lightcurves and $i$ '-band response. On the other hand, both Da08 and Po07 obtained the SN Ia rates at the highest redshift $(\mathrm{z} \sim 1.6)$. While both of these two results showed likely decreasing rates at the highest redshift, both of them also had large uncertainty due to small statistics. Hence we cannot get clear conclusion where the peak of a volumetric SN Ia rate is. According to galaxy studies, for example Hopkins \& Beacom (2006), the peak of cosmic star formation rates is at $\mathrm{z} \sim 2$-3. Compared to the peak of cosmic star formation rates, our results show the peak of SN Ia rates is at $\mathrm{z}>1.2$ and we estimate that the delay time of SNe Ia at high redshifts is $\lesssim 2-3$ Gyr. In order to discriminate whether SNe Ia having the short delay time are dominant or not crucially, it is necessary to observe more samples at $z \geqslant 1.4$.

\section{References}

Dahlen, T., et al. 2008, $A J, 136,1361$

Dilday, B., et al. 2008, ApJ, 682, 262

Frieman, J. A., et al. 2008, AJ, 135, 338

Hopkins, A. M., \& Beacom, J. F., 2006, ApJ, 651, 142

Hsiao, E. Y., et al., 2007, ApJ, 663, 1187

Mannucci, F. et al. 2006, MNRAS, 370, 773

Neill, J. D., 2006, AJ, 132, 1126

Poznanski, D., et al. 2007, MNRAS, 382, 1169

Totani, T., et al. 2008, PASJ, 60, 1327 